PROCEEDINGS OF THE

AMERICAN MATHEMATICAL SOCIETY

Volume 127, Number 7, Pages 2167-2173

S 0002-9939(99)04648-1

Article electronically published on March 1, 1999

\title{
GROWTH OF LEAVES IN TRANSVERSELY AFFINE FOLIATIONS
}

\author{
ROBERT A. WOLAK
}

(Communicated by Christopher Croke)

\begin{abstract}
In this note we give estimates for the growth of leaves in transversely affine foliations which depend on the properties of the affine holonomy group.
\end{abstract}

\section{INTRODUCTION}

Transversely affine foliations form a very interesting class of foliations and they appear naturally in differential topology and geometry and, for example, in the quantization theory. In the geometric prequantization the existence of a polarization plays an important role. Under certain natural assumptions such a polarization is defined by a pair of transversely affine foliations; cf. [12, 20]. One should also mention the role played by transversely affine foliations in "tissus feuilletés"; cf. $[6,19]$.

In spite of their relative popularity transversely affine foliations have not been studied in great detail and their properties are much less known than those of Riemannian and totally geodesic foliations.

In this short note we prove the following estimates for the growth type of a certain class of transversely affine foliations. The growth type of a foliation is a very interesting invariant which, in the case of Riemannian foliations, permitted a nice characterization of such foliations of polynomial growth; cf. [4]. However, the case of transversely affine foliations is more difficult. The main difficulty is the fact that in the case of Riemannian foliations the associated reduction of the bundle of transversely linear forms is compact, which is not the case for transversely affine foliations (of course assuming that the manifold $M$ is compact).

Our main results can be summarized in the following theorem.

Theorem 1. Let $\mathcal{F}$ be a complete transversely affine foliation on a compact manifold $M$. If the affine holonomy group of the foliation is nilpotent, then its structure algebra is nilpotent, the foliation has polynomial growth and $\operatorname{gr}(\mathcal{F}) \geq \delta(\underline{k})$ where $\delta(\underline{k})$ is the degree of nilpotency of the structure algebra of $\mathcal{F}$.

In the last section we present examples which show that Theorem 1 cannot be improved.

Received by the editors March 27, 1997 and, in revised form, July 30, 1997.

1991 Mathematics Subject Classification. Primary 57R30.

Key words and phrases. Transversely affine foliations, leaves, growth.

(C)1999 American Mathematical Society 


\section{Preliminaries}

Let $\mathcal{F}$ be a transversely affine foliation of codimension $q$ on a compact manifold $M$. $\mathcal{F}$ is developable, i.e., there exist a covering $\widehat{M}$ of $M$, a homomorphism $\alpha$ : $\pi_{1}(M) \rightarrow \operatorname{Aff}(E)$ and a global submersion $D: \widehat{M} \rightarrow E$ which is $\pi_{1}(M)$-equivariant. The group of the deck transformations of $\widehat{M}$ can be identified with $\Gamma=\operatorname{Im} \alpha$ - the affine holonomy group of $(M, \mathcal{F})$; cf. [9]. The lifted foliation $\widehat{\mathcal{F}}$ of $\widehat{M}$ is given by connected components of the fibres of $D$. The developing mapping $D$ can be neither surjective nor a locally trivial fibre bundle. If it is a locally trivial fibre bundle over $E$ the foliation $\mathcal{F}$ is called complete. Moreover, $\mathcal{F}$ is a $\nabla-G$-foliation with $\nabla$ being the canonical flat connection of $E$; cf. [21]. Thus, we have the following commutative diagram:

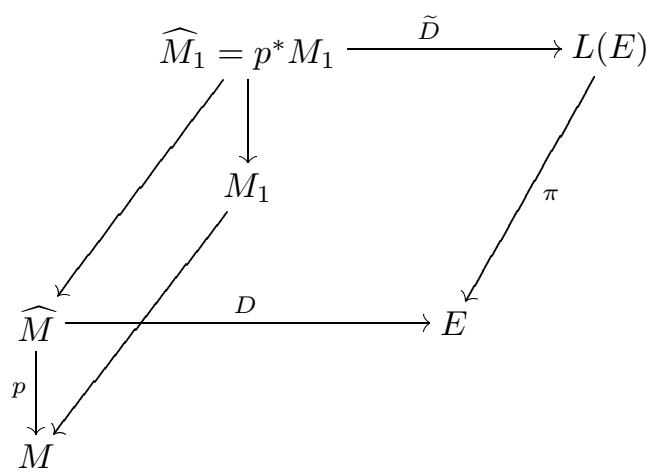

where $M_{1}=L(M, \mathcal{F})$ is the bundle of frames of the normal bundle of $\mathcal{F}$, i.e., the bundle of transverse frames of $(M, \mathcal{F})$; cf. [22, 15]. The bundle $M_{1}$ admits a canonical foliation $\mathcal{F}_{1}$ of the same dimension as $\mathcal{F}$ and whose leaves are coverings of leaves of $\mathcal{F} . \mathcal{F}_{1}$ is a developable foliation modelled on $L(E)$, the bundle of linear frames of $E$; thus it is an $\operatorname{Aff}(E)$-Lie foliation.

In [24] we have defined the structural (commutative) sheaf of a $\nabla-G$-foliation. This definition is based on Molino's definition of the structural sheaf of a Riemannian foliation; cf. $[14,15]$. We recall the definition and describe in detail this sheaf.

Let $\mathcal{C}_{1}$ be the sheaf of germs of foliated vector fields which commute with all global foliated vector fields of $\left(M_{1}, \mathcal{F}_{1}\right)$. As these vector fields must commute with the fundamental horizontal and vertical vector fields, locally, they are lifts of local infinitesimal transformations of the transversely projectable flat connection of $(M, \mathcal{F})$. Therefore, $\mathcal{C}_{1}$ defines a sheaf $\mathcal{C}$ of germs of foliated vector fields on $(M, \mathcal{F})$. We call $\mathcal{C}$ the structural sheaf of $\mathcal{F}$. Its stalks consist of germs of local foliated infinitesimal affine transformations of the transversely projectable flat connection and the Lie bracket endows them with the Lie algebra structure.

The lift $\widehat{\mathcal{C}}$ of the sheaf $\mathcal{C}$ to $\widehat{M}$ consists of germs of foliated vector fields of $(\widehat{M}, \widehat{\mathcal{F}})$ whose lifts to $\widehat{M}_{1}$, forming a sheaf $\widehat{\mathcal{C}}_{1}$, commute with all $\Gamma$-invariant global foliated vector fields. If $\mathcal{F}$ is complete $\widehat{C}_{1}$ projects to a sheaf $\mathcal{C}_{A}$ on $\operatorname{Aff}(E)$ whose elements commute with all global (left) $\Gamma$-invariant vector fields on $\operatorname{Aff}(E)$, thus with all $K=\bar{\Gamma}$-invariant vector fields. This means that the vector fields of the sheaf $\mathcal{C}_{A}$ must be tangent to the fibres of the $K$-fibre bundle $\operatorname{Aff}(E) \rightarrow K \backslash \operatorname{Aff}(E)=W$. Additionally, they must commute with the fundamental vertical vector fields $k^{*}$, 
$k \in \operatorname{Lie}(K)=\underline{k}$, of this bundle as well as with any vector field of the form $\sum f_{i} k_{i}^{*}$ where $k_{i} \in \underline{k}, f_{i} \in C^{\infty}(W)$. Thus, each stalk of $\mathcal{C}_{A}$ is isomorphic to the conjugated algebra $\underline{k}^{-}$. We call this Lie algebra the structure algebra of the transversely affine foliation $\mathcal{F}$. We have proved the following.

Proposition 1. Let $\mathcal{F}$ be a complete transversely affine foliation with the affine holonomy group $\Gamma$. Then its structural sheaf is a locally constant sheaf of Lie algebras whose stalk is isomorphic to the conjugated Lie algebra of $\operatorname{Lie}(K), K=$ $\bar{\Gamma} \subset \operatorname{Aff}(E)$.

For more properties of the structural sheaf and its relation to the closures of leaves see [24].

\section{THE PROOF OF THE MAIN THEOREM}

In [17] J. Plante studied in detail the growth of leaves. For foliations on compact manifolds he demonstrated that the growth type of the volume function of a leaf is the same as the growth type of the corresponding orbit of the holonomy pseudogroup. If we change a Riemannian metric on the manifold we get another volume function but, fortunately, any two Riemannian metrics on a compact manifold are equivalent (or the corresponding Riemannian manifolds are quasi-isometric). Therefore the corresponding volume functions have the same growth type. The holonomy pseudogroup in question is the pseudogroup associated to a finite foliated atlas of the foliated manifold $(M, \mathcal{F})$. Different finite foliated atlases define different pseudogroups but these pseudogroups are finitely equivalent in the sense of Haefliger; cf. [10, 11]. The corresponding orbits in finitely equivalent pseudogroups have the same growth type. The basic property that the growth type of the volume function of a leaf is the same as the growth type of the corresponding orbit of the holonomy pseudogroup is demonstrated in [17] using the fact that the plaques of the charts of the foliated atlas have the volume bounded both from below and above.

On an open manifold the growth type of the volume function of a leaf depends on a Riemannian metric or, to be precise, on the equivalence class of a Riemannian metric. Moreover, there is no relation between the growth type of the volume function of a leaf and the growth type of the corresponding orbit. However, if the foliated open manifold $(M, \mathcal{F})$ admits a finite foliated atlas whose plaques have the volume bounded both from below and above, then the growth type of the volume function of any leaf of $\mathcal{F}$ is equal to the growth type of the corresponding orbit of the pseudogroup defined by this foliated atlas. In this case we will say that the Riemannian metric is good.

Y. Carrière in his paper [4] studied in great deal the growth type of Riemannian foliations on compact manifolds. Using the Molino structure theorem one can reduce the field of interest to transversely Lie foliations. These foliations are developable and local transverse manifolds can be considered to be open subsets of the model Lie group $G$ and the generating transformations are restrictions of a finitely generated subgroup $\Gamma$ called the holonomy group of the foliation which is independent of the choice of a foliated atlas. Y. Carrière takes the union $V$ of these local transverse manifolds (in $G$ ) and the action of the group $\Gamma$ on $V$. The set $V$ is locally compact and the growth of the orbits of the pseudogroup generated on $V$ is dominated by the growth of the foliation; cf. [4]. 
Let $x \in V$, then let $\operatorname{gr}_{V}(\Gamma, x)$ denote the growth type of the $\Gamma$ orbit of $x$ in $V$. With this notation in mind, using the technique of Carrière's paper, it is not difficult to prove the following:

Lemma 1. Let $V$ be a relatively compact subset of $G$ and $V^{\prime}$ be any subset of $G$ with an open interior. If any $\Gamma$ orbit of a point in $V$ meets the set $V^{\prime}$, then $\operatorname{gr}_{V}(\Gamma, x) \leq \operatorname{gr}_{V^{\prime}}\left(\Gamma, x^{\prime}\right)$ for any point $x$ of $V$ and $x^{\prime}$ any point of the set $V^{\prime}$ belonging to the $\Gamma$ orbit of $x$.

This lemma leads us to a curious property of $G$-Lie foliations with dense leaves. It is well known (cf. $[8,15])$ that in this case the holonomy group has dense orbits. Let us compare the growth type of leaves of two such foliations. For suitable Riemannian metrics (on a compact manifold any choice is good) the growth type of a leaf (of the volume function) is the same as the growth type of the corresponding orbit of the holonomy group in an open subset of the Lie group $G$. Lemma 1 ensures the following:

Lemma 2. Let $\mathcal{F}$ be a $G$-Lie foliation with dense leaves of a compact manifold $M$ with the holonomy group $\Gamma$. If $\mathcal{F}_{1}$ is another $G$-Lie foliation with the holonomy group $\Gamma$ on a manifold $M_{1}$ with a good Riemannian metric, then $\operatorname{gr}(\mathcal{F}) \leq \operatorname{gr}\left(\mathcal{F}_{1}\right)$.

Now let us return to transversely affine foliations.

Since the foliation $\mathcal{F}$ is developable, we have the following estimate due to R. A. Blumenthal; cf. [3].

Proposition 2. Let $\mathcal{F}$ be a transversely affine foliation of a compact manifold $M$. Then $\operatorname{gr}(\mathcal{F}) \leq \operatorname{gr}\left(\pi_{1}(M)\right)$, or to be precise, $\operatorname{gr}(\mathcal{F}) \leq \operatorname{gr}(\Gamma)$.

Combining this result with the estimate of $\mathrm{H}$. Bass (cf. [2]) we get:

Corollary 1. Let $\mathcal{F}$ be a transversely affine foliation of a compact manifold $M$. If the fundamental group $\pi_{1}(M)$ is nilpotent, then $\operatorname{gr}(\mathcal{F}) \leq d(\Gamma)$.

This corollary together with Plante's theorem (cf. [17]) ensure that any transversely affine foliation with nilpotent, or virtually nilpotent, affine holonomy group admits a holonomy invariant measure; cf. [9].

The following result proved by Y. Carrière for Lie foliations is also valid for a larger class of foliations, among them for transversely affine foliations; cf. [4].

Proposition 3. Let $\mathcal{F}$ be a complete transversely affine foliation of a compact manifold $M$. Then the growth type of a leaf $L$ of $\mathcal{F}$ can be read as the local growth of the corresponding orbit of the affine holonomy group on $E$.

To get more information about the growth type of $\mathcal{F}$ we compare the growth type of leaves of $\mathcal{F}$ and $\mathcal{F}_{1}$. However, as $M_{1}$ is not compact, the growth type of a leaf is not well-defined; it depends on the choice of an adapted atlas; cf. [17]. Fortunately, the foliated manifold $\left(M_{1}, \mathcal{F}_{1}\right)$ has a class of adapted atlases which are particularly well-suited to the comparative study of the growth types of leaves of $\mathcal{F}$ and $\mathcal{F}_{1}$. We have in mind the atlases which are derived from adapted atlases of $(M, \mathcal{F})$; we call them preferred adapted atlases. If $M$ is compact, it can be easily shown that the growth type of a leaf of $\mathcal{F}_{1}$ does not depend on the choice of a preferred adapted atlas. Therefore, for any leaf $\widehat{L}$ of $\mathcal{F}_{1}$, we denote by $\operatorname{gr}(\widehat{L})$ the growth type of $\widehat{L}$ for any preferred adapted atlas of $\left(M_{1}, \mathcal{F}_{1}\right)$. Then:

Lemma 3. Let $\widehat{L}$ be a leaf of $\mathcal{F}_{1}$ covering a leaf $L$ of $\mathcal{F}$. Then $\operatorname{gr}(L) \leq \operatorname{gr}(\widehat{L})$. 
Proof. It is a simple consequence of the definition.

Considerations of the proof of Proposition 3 applied to the foliation $\mathcal{F}_{1}$ yield the following:

Lemma 4. The growth type of any leaf of $\mathcal{F}_{1}$ can be read as the growth type of the corresponding orbit of the action of the affine holonomy group restricted to the set $\pi^{-1}(U)$ where $U$ is a relatively compact subset of $E$.

Proof. The proof results from the considerations of Y. Carrière (cf. [4]) and the fact that we calculate the growth type relative to a preferred adapted atlas of $\left(M_{1}, \mathcal{F}_{1}\right)$.

Now we would like to find a lower bound for the growth of leaves of $\mathcal{F}$ depending on the structure of the affine holonomy group. Lemma 3 makes our task easier, as we can work with the Lie foliation $\mathcal{F}_{1}$. The closure of a leaf $\widehat{L}$ corresponds via $D$ to the orbit of the group $K=\bar{\Gamma} \subset \operatorname{Aff}(E)$. Let us assume that the affine holonomy group $\Gamma$ is nilpotent. In [23] we have proved that in this case $\Gamma$ and therefore $K$ must be unipotent. Thus, the connected component $K_{0}$ of $e$ in $K$ is a simply connected closed nilpotent Lie subgroup of $\operatorname{Aff}(E)$. The group $\Gamma_{0}=K_{0} \cap \Gamma$ is a finitely generated dense subgroup of $K_{0}$. Using the Malcev theorem (cf. [13, 18]), we can construct a Lie foliation $\mathcal{F}_{0}$ with dense leaves of a compact manifold for which the group $\Gamma_{0}$ is the holonomy group. In [4] Y. Carrière showed that in such a case $\operatorname{gr}\left(\mathcal{F}_{0}\right) \geq \delta\left(K_{0}\right)$ where $\delta\left(K_{0}\right)$ is the degree of nilpotency of $K_{0}$. It is not too difficult to notice that the leaves of such a Lie foliation have the same growth type since they have the same quasi-isometry type. Thus, the leaves have the same growth type. Putting these two facts together we learn that for any leaf $L$ of the foliation $\mathcal{F}_{0} \operatorname{gr}(L) \geq \delta\left(K_{0}\right)$. We have already remarked that the growth type of leaves of the foliation $\mathcal{F}_{1}$ can be read as the growth type of the orbits of $\Gamma$ relative to some open subset of $K$. As $\Gamma_{0} \subset \Gamma$, for any leaf $L_{1}$ of $\mathcal{F}_{1} \operatorname{gr}\left(L_{1}\right) \geq \operatorname{gr}\left(\mathcal{F}_{0}\right) \geq \delta\left(K_{0}\right)$. We know that many leaves of the foliation $\mathcal{F}$ have no holonomy; cf. [5]. As leaves of the foliation $\mathcal{F}_{1}$ are holonomy coverings of leaves of $\mathcal{F}$, then any leaf $L_{1}$ of $\mathcal{F}_{1}$ over a leaf $L$ of $\mathcal{F}$ without holonomy is diffeomorphic to it (to be precise, the natural projection is a diffeomorphism). It means that they have the same growth type. Summing up our considerations we have proved the following theorem; compare Proposition 3.1 of [4]. (The structure algebra of $\mathcal{F}$ is the conjugated algebra of $\left.\operatorname{Lie}\left(K_{0}\right).\right)$

\section{EXAMPLES}

To illustrate that Theorem 1 cannot be improved we provide two examples.

Let us consider the 3-dimensional solvable group $S_{1}$ (cf. [1]), which can be represented in the matrix form as follows:

$$
\left(\begin{array}{cccc}
e^{k z} & 0 & 0 & x \\
0 & e^{-k z} & 0 & y \\
0 & 0 & 1 & z \\
0 & 0 & 0 & 1
\end{array}\right)
$$

where $x, y$ and $z$ are real numbers and $k$ is a fixed real number such that $e^{k}+e^{-k}$ is an integer different from 2 . This group acts simply transitively on $R^{3}$ and admits a uniform discrete subgroup $\Gamma_{1}$. The manifold $\Gamma_{1} \backslash S_{1}=S_{1}\left(\Gamma_{1}\right)$ is an affine manifold 
which can be identified with the hyperbolic torus bundle $T_{4}^{3}, A \in \mathrm{SL}(2, Z), \operatorname{tr} A>2$; cf. [7].

Example 1. The projection $p_{2}: R^{3} \rightarrow R,(x, y, z) \mapsto(x, y)$, is $S_{1}$-equivariant for the natural actions of this group. Therefore, $p_{2}$ defines a complete transversely affine flow $\mathcal{F}^{2}$ of the compact manifold $S_{1}\left(\Gamma_{1}\right)$. The flow $\mathcal{F}^{2}$ is diffeomorphic to the flow of $T_{A}^{3}$ given by suspension of the matrix $A$. The growth type of the foliation $\mathcal{F}^{2}$ is linear since it is a flow with non-compact leaves. The affine holonomy group $\Gamma$ can be identified with a subgroup of $\operatorname{Aff}\left(R^{2}\right)$ generated by $A$ and the integer translations. Since $\Gamma$ is a discrete subgroup, the structure algebra of $\mathcal{F}^{2}$ is 0 dimensional. However, the group $\Gamma$ is solvable, but not nilpotent.

Example 2. The projection $p_{1}: R^{3} \rightarrow R,(x, y, z) \mapsto y$, is $S_{1}$-equivariant for the natural actions of this group. Therefore, $p_{1}$ defines a codimension 1 complete transversely affine foliation $\mathcal{F}^{1}$ of the compact manifold $S_{1}\left(\Gamma_{1}\right)$. The leaves of this foliation are dense, and only the leaves corresponding to rational numbers have holonomy. The foliation $\mathcal{F}^{1}$ is diffeomorphic to the proper foliation of $T_{A}^{3}$ corresponding to one of the eigenvalues of $A$; cf. [7]. The affine holonomy group of $\mathcal{F}^{1}$ is solvable, the structure algebra is isomorphic to $R$, but the leaves of $\mathcal{F}_{1}$ have exponential growth. In fact, since all leaves are dense, those corresponding to points of $T^{2}$ with rational coefficients are resilient. Hence, they must have exponential growth; cf. [7]. This implies that a complete transversely affine foliation with nilpotent structure algebra can even have the exponential growth type.

\section{REFERENCES}

1. L. Auslander, L. Green and F. Hahn, Flows on homogeneous spaces, Ann. of Math. Studies, No. 53, 1963. MR 29:4841

2. H. Bass, The degree of polynomial growth of finitely generated nilpotent groups, Proc. London Math. Soc. 25 (1972), 603-614. MR 52:577

3. R. A. Blumenthal, Transversely homogeneous foliations, Ann. Inst. Fourier 29 (1979), 143158. MR 81h:57011

4. Y. Carrière, Feuilletages riemanniens à croissance polynomiale, Comm. Math. Helv. 63 (1988), 1-20. MR 89a:57033

5. D. B. A. Epstein, K. C. Millet, and D. Tischler, Leaves without holonomy, J. London Math. Soc. 16 (1977), 548-552. MR 57:4193

6. E. Ghys, Flots transvesalement affines et tissus feuilletés, Mém. Soc. Math. France 46 (1991), 123-150. MR 92i:57026

7. E. Ghys and V. Sergiescu, Stabilité et conjugaison différentiable pous certains feuilletages, Topology 19 (1980), 179-197. MR 81k:57022

8. Cl. Godbillon, Feuilletages - Études géométriques, Progress in Math., 98, Birkhäuser, 1991. MR 93i: 57038

9. W. Goldman, M. Hirsch, and G. Levitt, Invariant measures for affine foliations, Proc. Amer. Math. Soc. 86 (1982), 511-518. MR 84a:57026

10. A. Haefliger, Pseudogroups of local isometries, Res. Notes in Math., Vol. 131, Pitman, Boston, 1985, 174-197. MR 88i:58174

11. A. Haefliger, Leaf closures in riemannian foliations, A Fète Topology, Academic Press, 1988, 3-32. MR 89c:57033

12. H. Hess, Connections on symplectic manifolds and geometric quantization, Lecture Notes in Math., vol. 836, Springer, 153-165. MR 82j:58056

13. A. I. Malcev, On a class of homogeneous spaces, Izv. Akad. Nauk SSSR Ser. Mat. 13 (1949), 9-32; Amer. Math. Soc. Transl. 9 (1951), 276-301. MR 10:507d; MR 12:589e

14. P. Molino, Feuilletages riemanniens sur les variétés compactes; champs de Killing transverses, C. R. Acad. Sci. Paris 289 (1979), 421-423. MR 80j:57026

15. P. Molino, Riemannian Foliations, Progress in Math., vol. 73, Birkhäuser, 1988. MR 89b:53054 
16. P. Molino, Orbit-like foliations, Proc. Geometric Study of Foliations, Tokyo, 1993, (T. Mizutani et al., ed.), World Scientific, 1994, 97-119. MR 97e:57030

17. J. Plante, Foliations with measure preserving holonomy, Ann. of Math. 102 (1975), 327-361. MR 52:11947

18. M. S. Rangunathan, Discrete Subgroups of Lie Groups, Springer, 1972.

19. M.-H. Rigal, Géométrie globale des systemes bihamiltoniens en dimension impaire, Thèse, Montpellier, 1995.

20. I. Vaisman, $d_{f}$-cohomology of Lagrangian foliations, Mh. Math. 106 (1988), 221-244. MR 89m:58084

21. R. A. Wolak, On $\nabla-G$-foliations, Rend. Cir. Mat. Palermo (2) 1984 Suppl. No. 6, 329-341. MR 86f: 53038

22. R. A. Wolak, Foliated and associated geometric structures on foliated manifolds, Ann. Fac. Sci. Toulouse Math. 10 (1989), 337-360. MR 97g:53036

23. R. A. Wolak, Transversely affine foliations compared with affine manifolds, Quart. J. Math. Oxford 41 (1990), 369-384. MR 91g:57031

24. R. A. Wolak, Closures of leaves in transversely affine foliations, Canad. Math. Bull. 34 (1991), 553-558. MR 93f:53022

Instytut Matematyki, Uniwersytet Jagielloński, Wl. Reymonta 4, 30-059 Kraków, POLAND

E-mail address: wolak@im.uj.edu.pl 